\title{
Evolution of a subtilisin-like protease gene family in the grass endophytic fungus Epichloë festucae Michelle K Bryant ${ }^{1}$, Christopher L Schardl ${ }^{2}$, Uljana Hesse ${ }^{2}$ and Barry Scott*1
}

\author{
Address: ${ }^{1}$ Institute of Molecular Biosciences, Massey University, Private Bag 11222, Palmerston North, New Zealand and ${ }^{2}$ Department of Plant \\ Pathology, University of Kentucky, Lexington, KY 40546-0312, USA \\ Email: Michelle K Bryant - m.k.bryant@massey.ac.nz; Christopher L Schardl - schardl@email.uky.edu; Uljana Hesse - uhesse@hotmail.com; \\ Barry Scott* - d.b.scott@massey.ac.nz \\ * Corresponding author
}

Published: 19 July 2009

BMC Evolutionary Biology 2009, 9:168 doi:10.1/86/147|-2148-9-168

This article is available from: http://www.biomedcentral.com/l47I-2/48/9//68

(c) 2009 Bryant et al; licensee BioMed Central Ltd.

This is an Open Access article distributed under the terms of the Creative Commons Attribution License (http://creativecommons.org/licenses/by/2.0), which permits unrestricted use, distribution, and reproduction in any medium, provided the original work is properly cited.
Received: 23 December 2008

Accepted: 19 July 2009

\begin{abstract}
Background: Subtilisin-like proteases (SLPs) form a superfamily of enzymes that act to degrade protein substrates. In fungi, SLPs can play either a general nutritive role, or may play specific roles in cell metabolism, or as pathogenicity or virulence factors.

Results: Fifteen different genes encoding SLPs were identified in the genome of the grass endophytic fungus Epichloë festucae. Phylogenetic analysis indicated that these SLPs belong to four different subtilisin families: proteinase K, kexin, pyrolysin and subtilisin. The pattern of intron loss and gain is consistent with this phylogeny. E. festucae is exceptional in that it contains two kexinlike genes. Phylogenetic analysis in Hypocreales fungi revealed an extensive history of gene loss and duplication.
\end{abstract}

Conclusion: This study provides new insights into the evolution of the SLP superfamily in filamentous fungi.

\section{Background}

Proteases catalyse the cleavage of polypeptides to oligopeptides or amino acids. In fungi, aspartic, cysteine, metallo-, serine and threonine proteases, as well as uncharacterised classes of proteases, have been identified, which have been classified according to the amino acid residues required for catalytic activity [1]. The serine proteases represent the most well known class, with two major superfamilies: subtilisin-like proteases (SLPs) and the trypsins. Both superfamilies use the same catalytic triad (Asp-His-Ser), which is thought to have evolved through convergent evolution [2].

Subtilisin-like proteases (SLPs) are ubiquitous in prokaryotes and eukaryotes. Six families of SLPs have been iden- tified [3]: subtilisins, proteinase K-type, thermitases, kexins, lantibiotic peptidases and pyrolysins. Phylogenomic analyses suggest three families of subtilisin-like proteases are present in fungi [4]. The first family, known as proteinase K-type, was first identified in fungi and named for its similarity to the widely known Tritirachium album proteinase $\mathrm{K}$ [5]. These proteases are generally characterised by the presence of subtilisin $\mathrm{N}$-terminal domain containing the propeptide, which is thought to act as an intramolecular chaperone to assist protein folding as well as inhibit enzyme activity $[6,7]$, and a catalytic peptidase S8 domain. Phylogenetic analyses suggest subfamilies 1 and 2 of this family contain secreted proteases, whereas subfamily 3 contains intracellular proteases localised to the vacuole [4]. The secreted proteases are thought to gen- 
erally play a nutritive role [8], but the vacuolar proteases appear to play a specialised role in the breakdown of autophagic bodies in the vacuole during autophagy, allowing recycling of macromolecules during nutrient starvation $[9,10]$.

The second family of SLPs identified in fungi is the kexins. Kexins have two major domains: a peptidase S8 catalytic domain, and a proprotein convertase domain. Kexin-type enzymes, first identified in the yeast Saccharomyces cerevisiae [11], play an important role in post-translational modification in eukaryotes. Secreted proteins in eukaryotes are often synthesized as preproproteins, which undergo two proteolytic processing events to become mature proteins. The prepeptide is normally removed by a signal peptidase in the endoplasmic reticulum [12]. The resulting proprotein is then transferred to the Golgi, where kexin-like enzymes cleave the propeptide to give the mature protein.

The third class was described as class I, or members of the subtilisin family [4]. Members of this family in fungi usually have inserts in the catalytic domain, and long carboxyl-terminal extensions, which are both characteristic of a family described as pyrolysins [3]. However, the pyrolysin family appears to be heterogeneous, with many different accessory domains. The class I subtilisins generally contain a protease-associated (PA) domain inserted into the catalytic domain [13], along with a DUF1034 domain (this study), which has an unknown function.

The subtilisin superfamily is an interesting case study for the evolution of multigene families. Gene duplication (and subsequent divergence) along with gene loss are important contributors to gene family evolution [14,15]. Gene loss can occur through either loss of gene function due to deleterious mutations or through complete deletion of the gene. There is evidence of extensive gene duplication and loss within the SLP family in fungal lineages, which has been correlated with differences in fungal lifestyles [4].

In this study, we examined the evolution of the SLP gene family from the endophytic fungus, Epichloë festucae. This fungus forms a mutually beneficial association with its host grass. We were interested in the gene family in this organism because of its plant symbiotic lifestyle and close taxonomic relationship to the insect pathogen Metarhizium anisopliae (both Clavicipitaceae), where SLPs are important as virulence factors. The availability of other fungal genomes, especially those from Fusarium, Nectria and Trichoderma spp. also allows comparisons of SLP family evolution in fungi through gene duplication, loss and divergence.

\section{Methods \\ Bacterial strains and plasmids}

E. coli strains were grown on LB agar plates, supplemented with ampicillin $(100 \mu \mathrm{g} / \mathrm{mL})$ where necessary.

\section{Fungal strains and growth conditions}

Cultures of Neotyphodium lolii strain Lp19 [16] and E. festucae strain Fl1 (ex cultivar SR3000) were grown and maintained as described previously $[17,18]$.

\section{Molecular biology techniques}

Fungal genomic DNA was isolated from freeze-dried mycelia using previously described methods $[19,20]$. Plasmid DNA was isolated and purified by alkaline lysis using either the Bio-Rad (Hercules, CA 94547, USA) Quantum plasmid miniprep or midiprep kits or the Roche (Roche Diagnostics N.Z., Ltd., Auckland, New Zealand) plasmid miniprep kit. Genomic DNA digests were transferred to positively charged nylon membranes (Roche) by capillary transfer [21] and fixed by UV crosslinking $(120,000 \mu \mathrm{J} /$ $\mathrm{cm}^{2}$ ) in an Ultraviolet cross-linker Cex-800 (UltraLum, Inc., Claremont, CA 91711, USA). Filters were probed with $\left[\alpha^{32} \mathrm{P}\right]$-dCTP $(3000 \mathrm{Ci} / \mathrm{mmol}$; GE Healthcare, Auckland, New Zealand) labeled probes (Additional File 1). DNA was labeled by primed synthesis with Klenow fragment using a High-Prime kit (Roche). Labeled probes were purified using ProbeQuant ${ }^{\mathrm{TM}}$ columns (GE Healthcare). Membranes were washed and hybridization signals detected by autoradiography as described previously [22].

\section{Gene cloning strategy}

Nine SLP genes were identified in E. festucae Fl1 either using sequences amplified from the closely related fungal species N. lolii Lp19 as described previously [39], or amplified from E. festucae Fl1 genomic DNA with degenerate primers (Additional Files 1 and 2). Probes for these genes were hybridized to both an E. festucae Fl1 genomic DNA library and a Southern blot containing restriction enzyme digests of E. festucae Fl1 genomic DNA. Screening of the genomic library identified clones containing DNA of the gene of interest. Southern hybridizations provided information about the restriction enzyme fragments containing the gene of interest, thus facilitating the subcloning of DNA fragments containing the desired gene. Six further SLP genes were identified in the genome of another E. festucae strain, E2368 (Additional file 1).

\section{Library screening}

Construction of the N. lolii Lp19 and E. festucae Fl1 genomic DNA libraries screened in this study was described previously $[23,24]$. The N. lolii Lp19 genomic DNA library was screened by plaque hybridization using standard methods [25]. For the E. festucae Fl1 genomic library prepared as described in [24], filters arrayed with DNA from 5088 independent ampicillin-resistant colo- 
nies at a $6 \times 6$ density with double offset (Australian Genome Research Facility, Melbourne, Australia) were screened by hybridization with radioactively labeled probes [25].

\section{Polymerase chain reaction and amplification conditions} Standard PCR amplifications of genomic DNA templates were carried out in $25 \mu \mathrm{L}$ reactions containing $10 \mathrm{mM}$ Tris- $\mathrm{HCl}, 1.5 \mathrm{mM} \mathrm{MgCl}_{2}$ and $50 \mathrm{mM} \mathrm{KCl} \mathrm{(pH} \mathrm{8.3),} 50 \mu \mathrm{M}$ of each dNTP, $200 \mathrm{nM}$ of each primer, $0.5 \mathrm{U}$ of Taq DNA polymerase (Roche) and $5 \mathrm{ng}$ of genomic DNA. The thermocycler conditions used were: $94^{\circ} \mathrm{C}$ for $2 \mathrm{~min} ; 30$ cycles of $94^{\circ} \mathrm{C}$ for $30 \mathrm{~s}, 60^{\circ} \mathrm{C}$ for $30 \mathrm{~s}$ and $72^{\circ} \mathrm{C}$ for $1 \mathrm{~min}$ per $\mathrm{kb}$, followed by a final step at $72^{\circ} \mathrm{C}$ for $5 \mathrm{~min}$.

\section{DNA sequencing}

DNA fragments were sequenced by the dideoxynucleotide chain-termination method [26] using Big Dye (Version 3) chemistry with oligonucleotide primers (Sigma Genosys, Castle Hill, Australia) specific for pUC118, pGEM-T Easy, and genomic sequences from N. lolii or E. festucae. Products were separated on either an ABI Prism 377 sequencer (Perkin Elmer, Waltham, MA 02451, USA) or an ABI 3730 analyzer (Applied Biosystems, Inc., Foster City, CA 94404, USA) at the Allan Wilson Centre Genome Service, Massey University, Palmerston North, New Zealand.

\section{Bioinformatic analyses}

Sequence data were assembled into contigs with SEQUENCHER (Gene Codes Corporation, Ann Arbor, MI 48108, USA) version 4.1 and analyzed and annotated using MacVector 7.2 (MacVector, Inc., Cary, NC 27519, USA). Sequence comparisons were performed at the National Center of Biotechnology Information (NCBI) site http://www.ncbi.nlm.nih.gov using the Brookhaven (PDB), SWISSPROT, GenBank (CDS translation), PIR and PRF databases employing algorithms for both local (BLASTX and BLASTP) and global (FASTA) alignments [27-29]. Potential open reading frames for SLP and unlinked non-SLP genes were identified using FGENESH, an HMM-based gene structural prediction using the Fusarium graminearum parameters http:linux1.softberry.coberry.phtml?topic=fgenesh\&grp= ograms\&sub group=gfind. There were some instances where SLPs were not annotated in genome sequences. TBlastN analysis, using a conserved region of the peptidase S8 domain as the query sequence, was used to identify all putative SLPs in genome sequences. Where additional SLP genes were identified they were included in the analysis. The presence of signal peptides was analyzed using SignalP3.0 [30]. Polypeptide alignments were performed using ClustalW [31] in MEGA4 [32]).

Phylogenetic analyses were conducted in MEGA4 [32]. The evolutionary history was inferred using maximum likelihood (PhyML)[33]. PhyML was run from the ATGC
Montpellier Bioinformatics platform at http://www.atgcmontpellier.fr/. The Newick files were imported into MEGA 4.0 [32] to view the trees which were saved in tif format. Sequence relationships were inferred using the Neighbor-joining (N-J) method [34]. The bootstrap N-J consensus tree inferred from 1000 replicates was taken to represent the evolutionary history of the taxa analyzed [35]. Branches corresponding to partitions reproduced in less than $50 \%$ bootstrap replicates were collapsed. The evolutionary distances were computed using the Poisson correction method [36] and are in the units of the number of amino acid substitutions per site. All positions containing alignment gaps and missing data were eliminated only in pairwise sequence comparisons (Pairwise deletion option).

Assignment of subtilisin-like proteases to different families was done on the basis of domain structure, similarity to other proteases and grouping in phylogenetic trees. Proteinase K type enzymes have propeptide and peptidase S8 domains. Subfamilies sf1, sf2 and sf3 were previously described [4]. Subfamilies sf4 and sf5 of this group were assigned on the basis of their phylogenetic grouping. The pyrolysins have an S8 domain, interrupted by a PA domain, and a DUF1034 domain. The two subfamilies within this group were assigned on the basis of previous work [4]. The OSPs have a peptidase S8 domain and distinct amino acid motifs unique to this family [37].

The following N. lolii and E. festucae sequences have been submitted to DDBJ/EMBL/Genbank databases: prtA and prtE (nucleotide accession EU515143/protein accessions ACB30133 and ACB30132), prtB (EF015481/ABK27194), prtC (FJ648718/ACN30265), prtD (EU515141/ ACB30128), prtF (EU515139/ACB30123), prtG (FJ648719/ACN30268), prtH (EU515135/ACB30121), prtI (EU515134/ACB30118), prtJ (FJ648720/ACN30270), prtK (EU515134/ACB30119), prtL (EU515136/ ACB30120), prtM (FJ648721/ACN30271), kexA (EU515138/ACB30122) and kexB (EU515140/ ACB30127).

The E. festucae strain 2368 genome sequence data are available at http://www.genome.ou.edu/fungi.html.

\section{Results and discussion}

The E. festucae genome contains fifteen members of the subtilisin superfamily

Using a combination of PCR amplification and whole genome analysis, 15 SLPs were predicted in the genome of the endophytic fungus E. festucae (Figure 1A; Additional file 3). prtA, prtE, prtB and $\operatorname{kex} B$ were initially identified in N. lolii, an asexual derivative of E. festucae [38]. prtD, prtF, prtG and prtH were identified in E. festucae strain Fl1 from sequences amplified with degenerate primers designed to an alignment of SLP sequences [39]. During this project, 


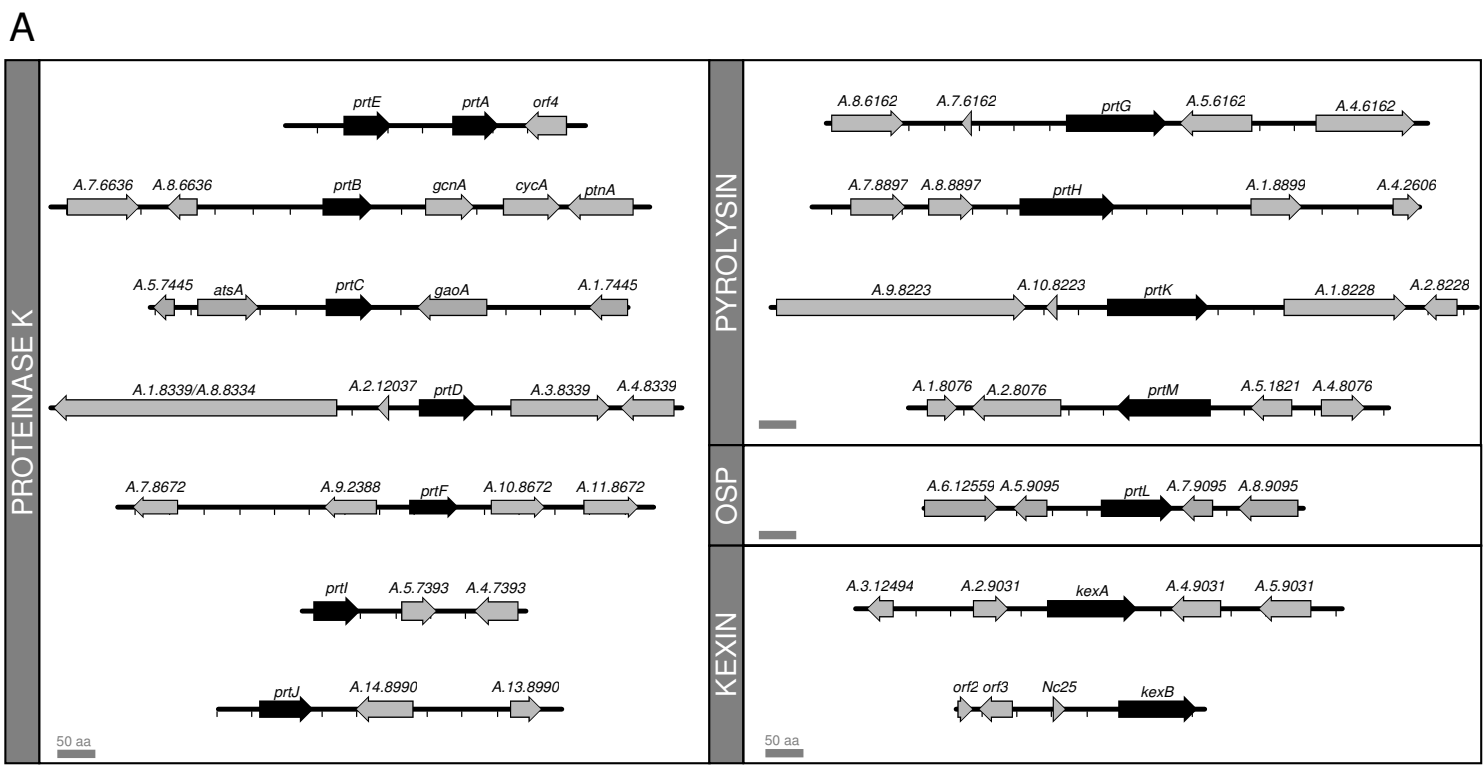

$\mathrm{B}$

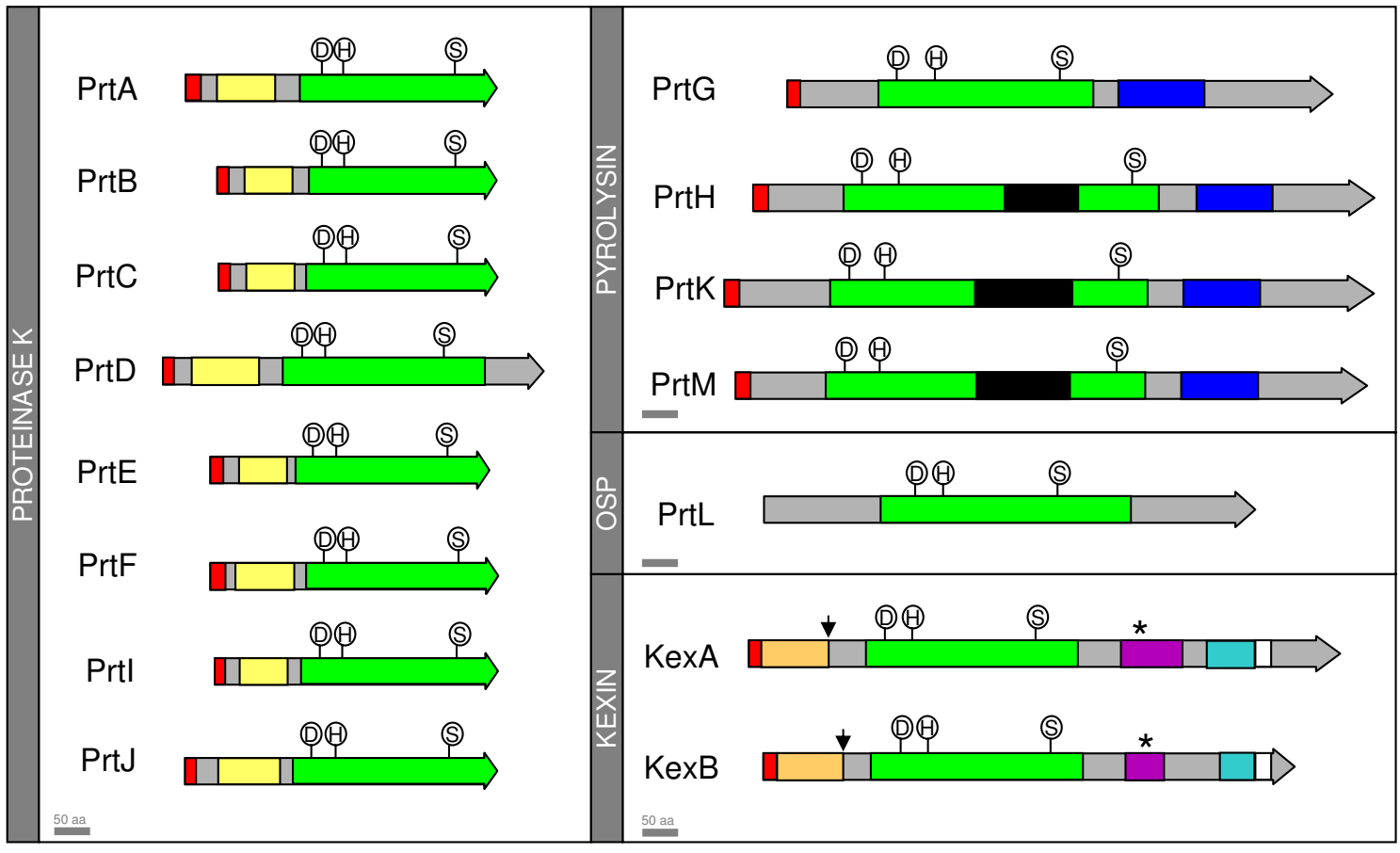

\section{Figure I}

Gene and protein structures of E. festucae FII SLPs. A. Genetic maps showing gene arrangement around the $E$. festucae SLP loci. Black arrows indicate the SLP genes, with non-protease genes indicated by light grey arrows. Gene names, where specific names have been assigned (in E. festucae FII), are shown above the genes. Systematic numbers assigned during annotation of the $E$. festucae E2368 genome refer to the remaining genes. Scale bars indicate a length of I kb. B. Domain organization of the $E$. festucae SLP proteins. Conserved catalytic triad residues are shown in circles above domain structures. The polypeptide is indicated in grey and colored blocks as follows: the signal peptide in red, subtilisin $\mathrm{N}$-terminal domains in yellow, peptidase S8 domains in green, PA domains in black, DUFI034 domains in blue, kexin propeptides in orange, P domains in purple, serine/ threonine-rich domains in teal and transmembrane domains in white. Conserved kexin autocatalytic cleavage sites are indicated by black arrows. Conserved RGD motifs in the kexin family are indicated by an asterisk. Scale bars indicate a length of 50 amino acid residues. 
the genome sequence for a closely related strain, E. festucae E2368, became available (C. Schardl, B. Roe, U. Hesse and J. W. Jaromczyk, unpublished). prtI, prtJ, prtK, prtL, prt $M$ and kexA were identified from the genome sequence of this E. festucae strain (Figure 1A). Direct sequencing of PCR products identified the corresponding genes in E. festucae Fl1. The predicted subtilisin-like protease (SLPs) genes ( $p r t A$ and $p r t B$ ) were identified in a genomic library from N. lolii [38] (Figure 1A). Probes used to screen the library were amplified with primers based on the Epichlö typhina At1 sequence [40]. Another predicted SLP-encoding gene, prtE, was identified directly upstream of the prtA gene (Figure 1A). Probing a genomic library also identified these three genes in E. festucae Fl1. Isolation of the prtB gene has been previously described [41]. Further predicted SLP genes were identified in the same library by screening with PCR products amplified using either primers designed to the E. typhina At 1 gene (prtC), or degenerate primers designed to conserved SLP sequences ( $p r t D$, prtF, prtG and prtH) (Figure 1A). The kexB gene, identified in N. lolii directly downstream of the Nc25 gene [42], was also identified by probing an E. festucae Fl1 genomic library (Figure 1A).

\section{The $E$. festucae subtilisin superfamily members represent} four different families

Sequence alignments and phylogenetic analysis showed that the predicted E. festucae SLPs grouped into four of the six different subtilisin families [3] (Figure 2). Eight genes
(prtA, B, C, D, E, F, I and $J$ ) encoded predicted proteins belonging to the proteinase $\mathrm{K}$ family (Figure $1 \mathrm{~B}$ ). While none of the E. festucae SLPs encoded by these prt genes has been tested for protease activity, the PrtC homologue from E. typhina, At1, has been shown to have subtilisinlike protease activity [40]. Three subfamilies of proteinase K-like enzymes have been previously identified, two of which are extracellular and one that is vacuolar in localization [4]. The predicted prtB, $C, E$ and $I$ gene products belong to subfamily 1 , while the predicted prtA and $F$ gene products belonged to subfamily 2 . As expected based on its isolation with degenerate primers designed to members of the vacuolar subfamily, the predicted prtD gene product belongs to subfamily 3. Although the predicted prtJ gene product was obviously in the proteinase $\mathrm{K}$ family, it did not belong to any of the known classes. Instead, it was found in a new subfamily of proteinase K-type enzymes we propose to call subfamily 4 (Figures 2 and 5; Additional file 4). Many other Sordariomycete fungi, as well as some Orbiliomycetes, contain members of this subfamily.

Four genes (prtG, $H, K$ and $M$ ) are predicted to encode proteases from the previously described class I or subtilisin family [4]. In fungi, these proteases contain a peptidase S8 domain, generally interrupted by a proteaseassociated (PA) domain, sometimes followed by a domain of unknown function (DUF1034) at the carboxylterminus. With the exception of PrtG, which is lacking the
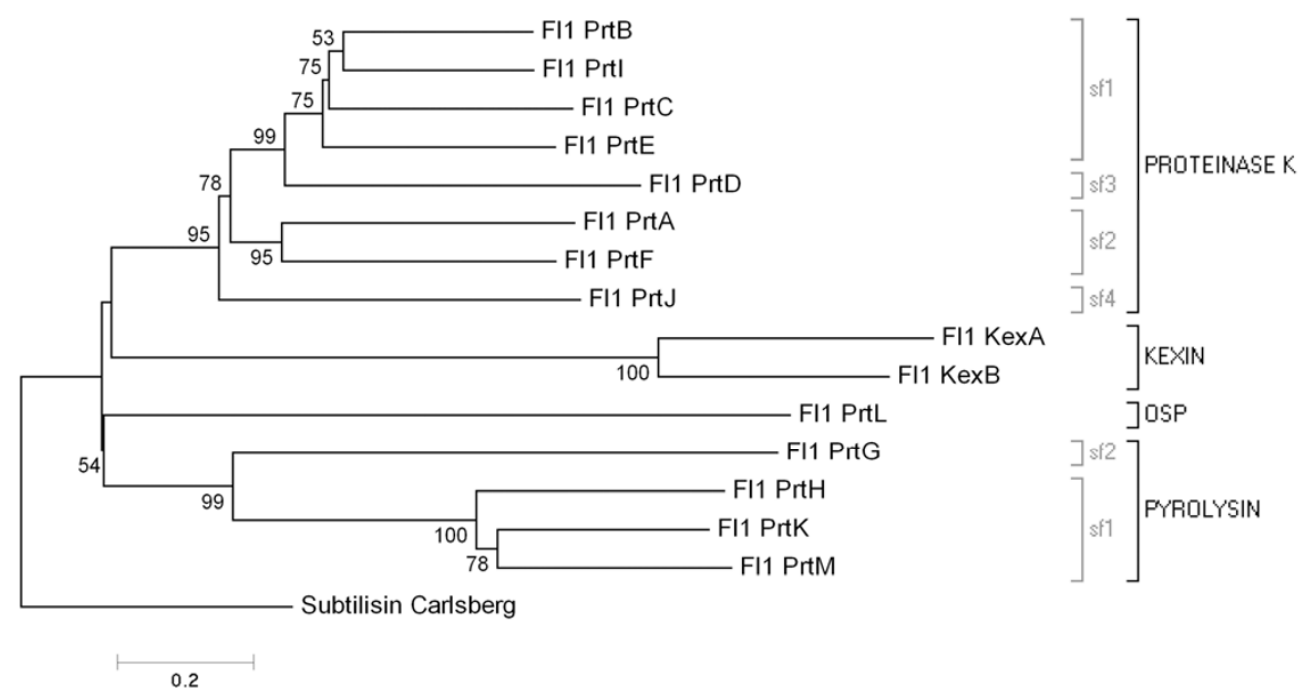

Figure 2

Evolutionary relationships of $\boldsymbol{E}$. festucae FII SLPs. Neighbor joining analysis determined phylogenetic relationships. The phylogram is rooted using the Bacillus subtilis subtilisin Carlsberg protein (accession P00780) as an outgroup. The percentages of replicate trees in which the associated taxa clustered together in the bootstrap test ( 1000 replicates) is shown next to the branches. The tree is drawn to scale, with branch lengths in the same units as those of the evolutionary distances used to infer the phylogenetic tree. SLP families and subfamilies are indicated by black or grey square brackets respectively. 
PA domain insert, the E. festucae pyrolysin-like enzymes have this domain structure (Figure $1 \mathrm{~B}$ ). Based on phylogenetic analyses, Hu and St Leger proposed that this family, which they called subtilisin class I, contained two subfamilies of ascomycete proteins [4]. Phylogenetic analysis suggested the predicted $p r t H, K$ and $M$ gene products belonged to subfamily 1 , whereas the predicted prt $G$ product belonged to subfamily 2 (Figures 2 and 5; Additional file 5).

These gene products may be part of the pyrolysin family, in which proteins have long carboxyl-terminal extensions and large insertions in the catalytic domain [3]. In the basidiomycete wood rot fungus Pleurotus ostreatus, a fungal pyrolysin of this type cleaves and activates other proteases, which in turn cleave and activate laccase isoenzymes, in an activation cascade [13]. It remains to be determined if these proteases play similar roles in other fungi.

Both the PA domain and DUF1034 domains have unknown functions. The PA domain may play a role in determining substrate specificity [43], as PA domain insertion in the catalytic peptidase S8 domain may interfere with the substrate reaching the active site [13]. However, in the C5a peptidase (Streptococcus spp.), which has a similar domain structure to the fungal pyrolysins, structural analysis suggests the PA domain is not in a position where it affects substrate specificity [44]. While the function of the DUF1034 domain (PFAM accession PF06280) is unknown, this domain is often present in bacterial and plant SLPs.

The predicted kexA and kexB gene products belong to the kexin family, which contains enzymes with a specialized role in proprotein processing in the secretory pathway (Figure 5; Additional file 6). The E. festucae genome is unusual among ascomycetes in that it contains two kexinlike genes. Like other kexins, the predicted kexA and kexB gene products both contain putative peptidase S8 and proprotein convertase $(\mathrm{P})$ domains [3] (Figure 1B). The putative $P$ domain in both the predicted KexA and KexB proteins contained an RGD motif, which is conserved in A. nidulans, A. niger and mammalian furins, but not $S$. cerevisiae Kex2p [45]. The predicted KexA and KexB proteins also contained putative serine/threonine-rich and transmembrane regions downstream of the putative P domain, which are conserved in other kexins. In S. cerevisiae, the propeptide of the KEX2 gene product is removed by autocatalysis, with cleavage on the carboxyl-terminal side of a Lys-Arg site [46]. Putative propeptide cleavage sites appeared in both the predicted KexA (Lys112-Arg113) and KexB (Arg112-Arg113) proteins.

The predicted prtL gene product did not belong to any of the subtilisin families previously described in fungi. How- ever, sequence comparisons and phylogenetic analysis suggested it was highly similar to a group of proteases called the oxidatively stable proteases (OSPs) [37] (Figures $1 \mathrm{~B}$ and 5; Additional file 7). The OSPs form a subfamily within the subtilisin family [47]. Like other OSPs, the predicted prtL gene product contained many insertions in the peptidase S8 catalytic domain relative to subtilisin Carlsberg, as well as a carboxyl-terminal extension of unknown function that may be required for structural integrity of these enzymes.

\section{Intron conservation}

Intron gains and losses are important in the evolution of gene families $[48,49]$. Intron position was examined in the members of the Fl1 SLP genes (Figure 3). Intron positions were predicted by the gene structure prediction program FGENESH. Sequencing of cDNA amplified from the prtA, prtB, prtE and kexB gene products validated the FGENESH predictions for these genes. In the proteinase $\mathrm{K}$ family, all of the genes except prtJ had a first intron at a conserved position (intron position 1, Figure 3A), suggesting all of these genes were derived from a common ancestral gene. A second intron position was also conserved in prtB, $C$ and $E$ (intron position 2, Figure $3 A$ ), while a third intron position was conserved in $\operatorname{prt} B, C$ and $I$ (intron position 7, Figure 3A) Comparison of the prtI gene with the orthologous $\operatorname{pr} 1 A$ gene from $M$. anisopliae revealed that the second of three introns in the pr1A gene appears to have been lost from prtI (missing intron position 2, Figure 3A). The loss of this intron, and those described subsequently (see below) appears to be due to complete deletion of the intron as there are no apparent relics of the intron left behind. Consequently, the reading frame of the gene is not altered. The prtF gene, which is a homologue of the M. anisopliae pr1J gene, contained two introns (intron positions 1 and 5, Figure 3A). In the $M$. anisopliae strains where pr1J had been sequenced previously, it was suggested an intron was inserted in two strains, rather than the other strain losing an intron [50]. However, the prtF gene contains both introns in the same conserved positions as pr1J, suggesting that where pr1J homologues contained only one intron, this situation has arisen by intron loss. Intron position was not conserved in prt $J$ relative to the other E. festucae genes (intron positions 3, 6, and 7, Figure 3A), but was conserved with closely related genes such as FGSG_09382 (F. graminearum).

Introns were in conserved positions in three of the four pyrolysin-type genes (Figure 3B). prtH, $K$ and $M$ have a first intron at a conserved position (intron 1, Figure $3 \mathrm{~B}$ ), with a second conserved intron in $p r t H$ and $p r t K$, but apparently lost in prtM (intron position 4, Figure 3B). The prtG gene did not share any common introns with other Fl1 pyrolysin-type genes (intron positions 2, 3, 5-14; Figure $3 \mathrm{~B}$ ). Both of the kexin type genes, $\operatorname{kex} A$ and $k e x B$, share common introns towards the $3^{\prime}$ end of the coding 

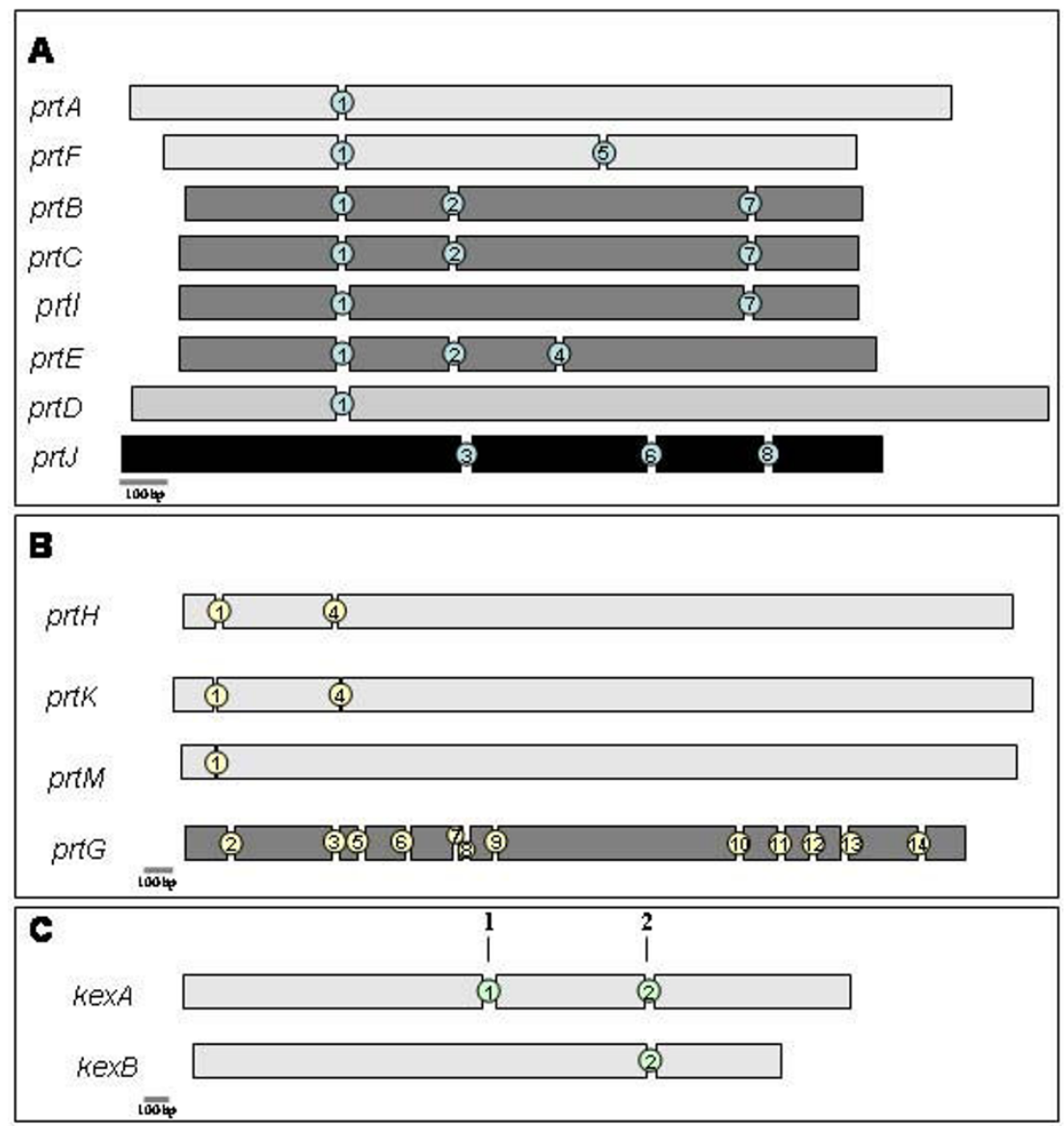

\section{Figure 3}

Exon-intron structures of $E$. festucae SLPs. Exon-intron structures of the $E$. festucae SLP like genes: proteinase K (A), fungal pyrolysins (B), and kexins (C). Each exon is indicated by a separate rectangle, with spaces between rectangles representing introns. Intron positions found in each family are indicated by numbers between exons. All scale bars indicate a length of $100 \mathrm{bp}$.

sequence (intron position 2, Figure 3C). However, the kexA gene contained an additional intron in the middle of the coding sequence (intron position 1, Figure 3C), which kexB did not share. This additional intron is conserved in Fusarium oxysporum, Fusarium verticillioides and Trichoderma spp., but is not found in other fungal species. This suggests that the additional intron has been gained in the Hypocreales lineage. A lack of introns in the prtL gene excluded it from this analysis.

\section{Synteny analysis}

Sequence analysis revealed that four E. festucae Fl1 SLP genes shared microsynteny with the related fungi Fusarium graminearum, Trichoderma reesei, and in some cases Neurospora crassa (Figure 4). These genes were kexA, prtD, prtG and prtK. The kexA (kexin-like) and prtD (vacuolar SLP) genes have highly specialized functions within the cell, which may suggest that conservation of the region around these genes is linked to their conserved function, a hypothesis supported by an analysis of regions of conserved microsynteny between the genomes of Magnaporthe grisea and Neurospora crassa [51] and other regions of the E. festucae genome [24,52]. However, the role of the pyrolysin-like enzymes in fungal cells is not well understood, so it is unclear what significance the synteny of the prtG and prtK genes may have.

\section{Comparison of the E. festucae SLP family with those from related fungi}

In order to determine how the predicted SLP family in E. festucae compares with those in other fungal species, a comprehensive survey of these genes in fungal genomes was performed. Numbers of predicted SLP-encoding genes varied from a minimum of three (Aspergillus nidulans and Aspergillus oryzae), to a maximum of 32 (Trichoderma virens) (Additional file 8 ). The wide variation 


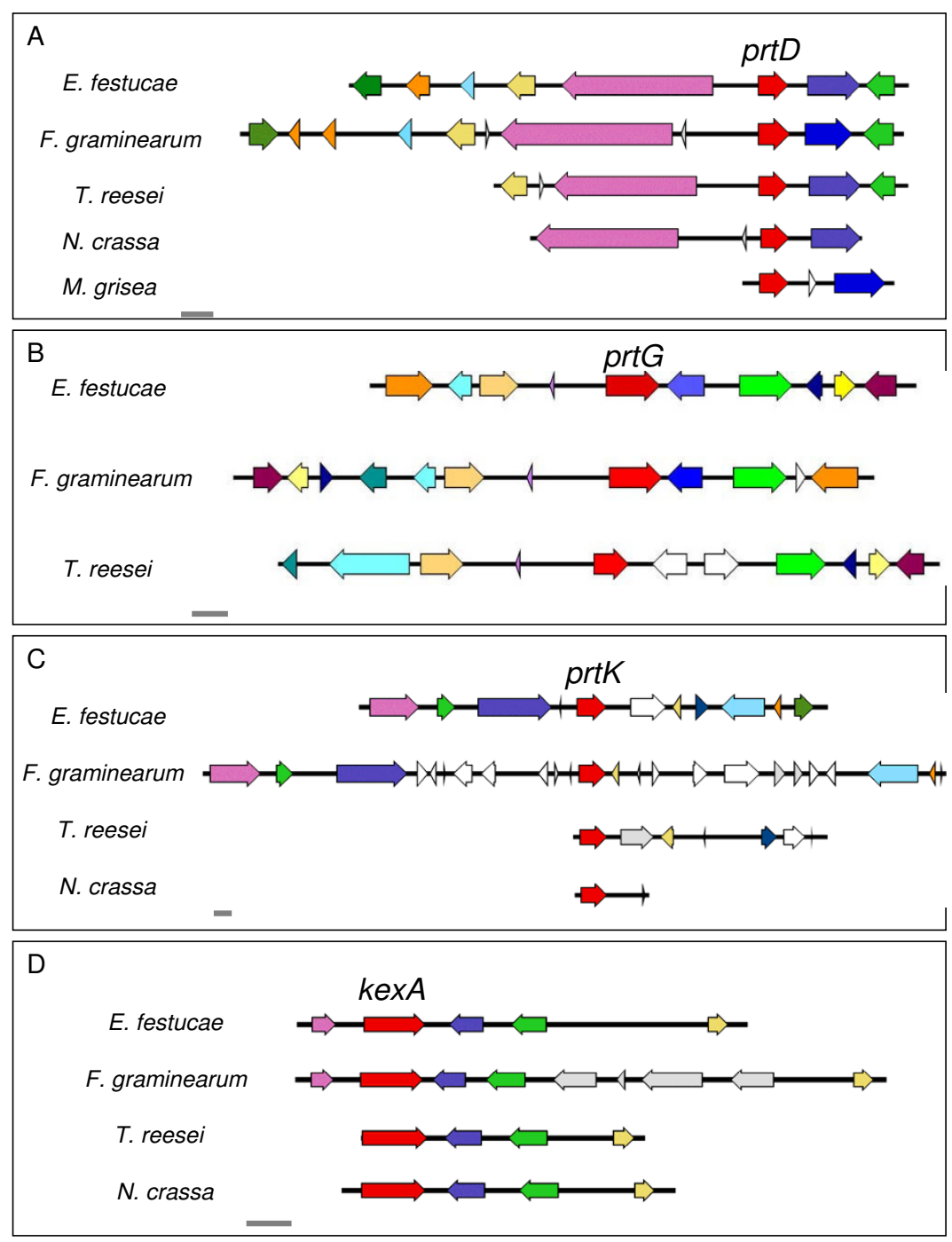

\section{Figure 4}

E. festucae SLP genes sharing conserved microsynteny with other fungal species. Conserved microsynteny at the prtD (A), prtG (B), prtK (C). and kexA (D) loci. SLP genes are shown in red. Non-orthologous genes are shown in grey or white. Orthologous genes are indicated by colours other than red, grey or white. All scale bars indicate a length of $2 \mathrm{~kb}$.

suggested that the processes of gene duplication and loss have been important in the evolution of this gene family in fungi.

The numbers of predicted SLP genes did not correlate with genome size. For instance, Aspergillus clavatus four SLPencoding genes were inferred in a $35 \mathrm{Mb}$ genome, whereas Nectria haematococca had 29 SLP-encoding genes inferred in a $40 \mathrm{Mb}$ genome. Generally, saprotrophic fungi had fewer predicted SLP-encoding genes than the phytopathogenic fungi (Additional file 8); however, a noticeable exception to this trend was the fact that the phytopathogenic Botrytis cinerea and Sclerotinia sclerotiorum species both had only four SLP genes each, numbers comparable to many of the saprotrophic Aspergillus spp.

The types of SLP-encoding genes found in fungal genomes were also classified. Previously, only three classes of SLPs had been identified in fungi: proteinase $\mathrm{K}$, kexin, and a subtilisin-like class. Phylogenetic analyses showed that there appeared to be other SLP classes in fungi. The class first represented by the $M$. anisopliae Pr1C enzyme, described as subtilisin class I [4], contains several unusual features. Unlike most subtilisins, the members of this group often contain an insertion of a protease-associated domain in the subtilisin catalytic domain, and a domain of unknown function, DUF1034, in the carboxyl-termi- 
nus. There has been a suggestion that this class of SLPs are pyrolysins [13], which have large insertions and/or carboxyl terminal extensions [3].

\section{Evolution of the SLP gene family in the Hypocreales}

Due to the number of Hypocreales genomes sequenced, this group is a good model to study the evolution of gene families, especially for inferring numbers of ancestral genes and patterns of gene gain and loss. Seven genomes are available within this group: F. graminearum, F. oxypsorum, F. verticillioides, N. haematococca, T. reesei, Trichoderma virens and E. festucae. Along with data derived from expression studies in M. anisopliae, this enabled comparisons to be made between SLP-encoding genes in this group.

The first obvious difference between the genome strains was the number of predicted SLP-encoding genes. E. festucae had the lowest number of SLP-encoding genes, with just 15 genes present in the genome. This was about half the number found in strains such as F. graminearum and T. virens. These differences are presumably due to either gene loss in E. festucae, or gene duplication in strains with high numbers of SLPs. To test this theory, phylogenetic analysis was used to assess the relationships between the Hypocreales SLPs (Figure 5, Additional file 9).

In the proteinase $\mathrm{K}$ family, a complicated pattern of gene duplication and loss has occurred. Analyses suggested that the common ancestor of the Hypocreales must have contained at least six proteinase $\mathrm{K}$ type genes, two of which must have belonged to subfamily 1 . In E. festucae Fl1, the prtE and prtB/prtC/prtI group represent these genes. For the prtE homologues, a gene duplication event seems to have occurred in the $F$. graminearum $/ F$. oxysporum $/ F$. verticillioides lineages, but not in N. haematococca. In the case of the $F$. oxysporum and F. verticillioides spp. prtE homologues, one of the duplicated genes appeared to have lost functionality due to a premature stop codon (FVEG_03737 and FOXG_05860). The prtE homologues appear to have been lost from the Trichoderma spp. lineages.

The prtB/prtC/prtI group was represented in the Trichoderma spp. as a single gene, but was lost in the Fusarium spp. and N. haematococca. The gene ancestral to prtB/prtC/prtI appeared to have undergone extensive gene duplication in the Clavicipitaceae lineages (E. festucae and M. anisopliae) to produce prtC (Fl1)/pr1G (M. anisopliae), prtB/pr1I, and $\operatorname{prtI} / \operatorname{pr} 1 A$, along with a further duplication to give the $M$. anisopliae pr1B gene. Pr1A and related enzymes in M. anisopliae are thought to act as virulence factors [53], which are effectively in an "arms race" with the protease inhibitors of the insect immune system, so these proteases may have duplicated and diversified to allow the fungi to colonize new hosts. This history of gene duplications is supported by intron position (Figure 3 ), with the prtB, prtC,
prtE and prtI genes all sharing two common introns, with a third intron shared by prtB, prtC and prtI.

In proteinase $\mathrm{K}$ subfamily 2, there also appears to be three ancestral genes, represented by the prtF, prtA and FGSG_03315 (F. graminearum) genes. E. festucae appears to have lost genes from the FGSG_03315 group, which is present in one copy in the $T$. reesei and $T$. virens genomes, but in two copies in the N. haematococca and Fusarium spp. genomes, suggesting that gene duplication has taken place in the common ancestor of these species. In the prtF homologues, a single gene is present in all the lineages except the Trichoderma spp., where gene loss appears to have occurred, and F. graminearum, where a gene duplication event appears to have taken place. The prtA homologues may have arisen from duplication of one of the other subfamily 2 genes, as it only contains genes from Fusarium spp., N. haematococca and the Clavicipitaceae fungi, E. festucae and M. anisopliae. While the E. festucae and $M$. anisopliae genomes contain only a single copy of this gene, two subsequent gene duplications have taken place in the Fusarium spp. and N. haematococca species to give three prtA-like genes for this group.

An unusual case in the M. anisopliae genome is the pr1E and $\operatorname{pr} 1 F$ genes, which are located in tandem. Bagga et al [54] suggest that the ancestor of $M$. anisopliae contained pr1D and pr1J genes, duplication and divergence of a pr1Jlike ( $p r t F$ group) gene giving rise to a pr $1 F$-likegene, which subsequently reduplicated to give the $\operatorname{pr} 1 \mathrm{E}$ and $\operatorname{pr} 1 \mathrm{~F}$ genes within the Metarhizium genus. The pr1E gene appears to have arisen by tandem duplication of the pr $1 F$ gene within M. anisopliae, after divergence of the f. spp. anisopliae and acridum [54].

Proteinase K subfamily 3, containing the specialized vacuolar proteases, is represented by a single gene in all of the Hypocreales strains, suggesting the common ancestor contained this gene. As described earlier, this study revealed the presence of new subfamilies within the proteinase $\mathrm{K}$ family. The prtJ gene is representative of the new subfamily 4 , which is present in a single copy in all of the Hypocreales strains except $M$. anisopliae, where the genome is unsequenced. This gene may be present in the M. anisopliae genome, but was undetected during expression studies used to identify SLPs in this organism. The presence of this gene in the Hypocreales genomes (Figure 5 ) as well as in other Sordariomycete genomes (Additional file 4), suggest this gene was present in the common ancestor.

The newly proposed subfamily 5 , characterized by the CHGG_10086 gene from Chaetomium globosum, has patchy taxonomic distribution within the Hypocreales, being only found in the F. oxysporum and F. verticillioides genomes (Figure 5; Additional file 4). This gene appears to 


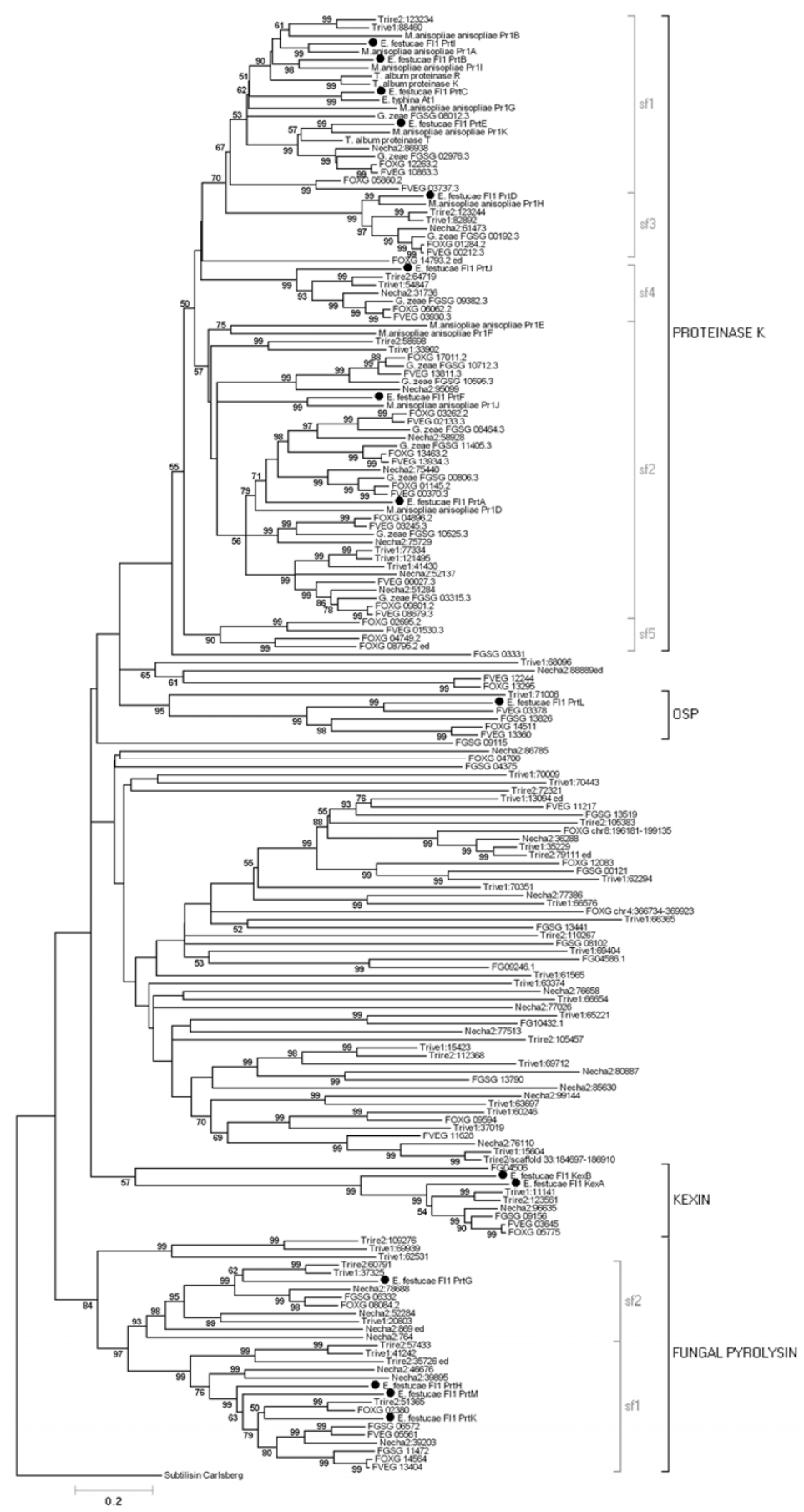

\section{Figure 5}

Evolutionary relationships of Hypocreaceae SLPs based on neighbor joining analysis. The phylogram is rooted using the Bacillus subtilis subtilisin Carlsberg protein (accession P00780) as an outgroup. The percentage of replicate trees in which the associated taxa clustered together in the bootstrap test (1000 replicates) are shown next to the branches. The tree is drawn to scale, with branch lengths in the same units as those of the evolutionary distances used to infer the phylogenetic tree. SLP families and subfamilies are indicated by black or grey square brackets respectively. E. festucae sequences are marked by black circles. Note: FOXG_I4793 does not group with sf3 (the vacuolar SLPs) or sf4 (characterized by FGSG_09382) but falls into a group by itself. Additional analysis showed it groups with proteases from $P$. anserina and $N$. fischeri. 
have undergone at least one duplication event in the ancestor of these two species to give two genes, followed by another duplication in F. oxysporum to give a third gene. However, it is interesting to note that in F. verticillioides, frameshifts due to base insertion or deletions have created genes that appear to encode non-functional proteins (FVEG_01530 and FVEG_03386), whereas one of the F. oxysporum genes, FOXG_02695, also appears to have undergone a similar frameshift.

Gene duplication and gene loss was studied in the fungal pyrolysin family. For subfamily 2 , a single representative was found in each of the Hypocreales genomes, except $M$. anisopliae (possibly due to not having the complete genome sequence). This subfamily was previously shown to have undergone extensive gene duplication in the more distantly related M. grisea [4] (Additional file 5). In subfamily 1, gene duplication or loss may have taken place multiple times. All of the Hypocreales genomes contained at least one prtK-like gene, with multiple copies in the Fusarium spp. and E. festucae.

In the kexin family, all Hypocreales strains (except M. anisopliae) have at least one kexin gene (Figure 5; Additional file 6). This gene appears to have been duplicated in E. festucae. The differences between the sequences appear to indicate divergence of kexB from kexA.

The prtL gene, which represents the OSP subfamily, was present in most of the Hypocreales, except $T$. reesei, where the gene appears to have been lost, and in F. verticillioides, where the gene appears to have been duplicated (Figure 5; Additional file 7).

A complicating factor in assessing the evolution of the Hypocreales SLP superfamily is the presence of many sequences with lower SLP similarity in Trichoderma, $N$. haematococca and Fusarium spp (Figure 5). These sequences, which generally encode large proteins with a peptidase S8 domain characteristic of SLPs, were not present in the E. festucae genome, suggesting they may have been lost from these strains. An interesting feature of some of these proteins was the presence of ankyrin repeats in the amino terminus of the protein, with a peptidase $S 8$ domain in the carboxyl terminus (e.g. FGSG_04375 from F. graminearum). The role of these proteins within the cell is unknown, but potentially the ankyrin repeats, which are involved in protein-protein interactions [55], could target SLP activity towards particular protein substrates.

\section{Conclusion}

In this study, we aimed to study the evolution of the SLP gene family in E. festucae. Fifteen predicted SLP genes were present in the E. festucae genome, representing four different SLP families. New subfamilies within the proteinase K family were identified, as well as a new family, the oxidatively stable proteases previously thought to be present only in bacteria. Phylogenetic studies showed that many gene duplications and loss events have occurred during evolution of the SLP gene family within the Hypocreales.

\section{Authors' contributions}

MKB carried out the E. festucae Fl1 DNA sequencing, bioinformatics and drafted the manuscript. BS participated in the design and coordination of the study and helped to draft and revise the manuscript. CLS assisted with the phylogenetic analysis and provided the E. festucae E2368 sequence. All authors read and approved the final manuscript.

\section{Additional material}

\section{Additional file 1}

Table describing list of probes. Probes used for Southern hybridization and $\mathrm{E}$. festucae genomic library screening.

Click here for file

[http://www.biomedcentral.com/content/supplementary/14712148-9-168-S1.doc]

\section{Additional file 2}

Table of primer sequences. Sequences of primers used to amplify prt genes.

Click here for file

[http://www.biomedcentral.com/content/supplementary/14712148-9-168-S2.doc]

\section{Additional file 3}

Table showing Bioinformatic analysis of E. festucae subtilisin-like protease genes. Bioinformatic analysis of E. festucae strain Fl1 subtilisinlike protease genes.

Click here for file

[http://www.biomedcentral.com/content/supplementary/14712148-9-168-S3.doc]

\section{Additional file 4}

Evolutionary relationships of fungal proteinase $K$ family genes based on PhyML analysis. The phylogram (drawn to scale) is rooted using the Bacillus subtilis subtilisin Carlsberg protein (accession P00780) as an outgroup. Numbers at branches indicate the percentage of 1000 bootstrap replicates that supported each branch. E. festucae sequences are marked by black circles.

Click here for file

[http://www.biomedcentral.com/content/supplementary/14712148-9-168-S4.jpeg]

\section{Additional file 5}

Evolutionary relationships of fungal pyrolysin genes based on PhyML analysis. The phylogram (drawn to scale) is rooted using the Bacillus subtilis subtilisin Carlsberg protein (accession P00780) as an outgroup. Numbers at branches indicate the percentage of 1000 bootstrap replicates that supported each branch. E. festucae sequences are marked by black circles.

Click here for file

[http://www.biomedcentral.com/content/supplementary/1471-

2148-9-168-S5.jpeg] 


\section{Additional file 6 \\ Evolutionary relationships of fungal kexin genes based on PhyML analysis. The phylogram (drawn to scale) is rooted using the Bacillus subtilis subtilisin Carlsberg protein (accession P00780) as an outgroup. Numbers at branches indicate the percentage of 1000 bootstrap replicates that supported each branch. E. festucae sequences are marked by black circles. \\ Click here for file \\ [http://www.biomedcentral.com/content/supplementary/1471- 2148-9-168-S6.jpeg] \\ Additional file 7 \\ Evolutionary relationships of fungal OSP genes based on PhyML anal- $\gamma$ sis. The phylogram (drawn to scale) is rooted using the Bacillus subtilis subtilisin Carlsberg protein (accession P00780) as an outgroup. Numbers at branches indicate the percentage of 1000 bootstrap replicates that sup- ported each branch. E. festucae sequences are marked by black circles. Click here for file \\ [http://www.biomedcentral.com/content/supplementary/1471- 2148-9-168-S7.jpeg] \\ Additional file 8 \\ Table showing taxonomic distribution of subtilisin like proteases. Tax- onomic distribution of subtilisin-like proteases in fungal genomes. \\ Click here for file \\ [http://www.biomedcentral.com/content/supplementary/1471- 2148-9-168-S8.doc] \\ Additional file 9 \\ Table on distribution of subtilisin like proteases. Distribution of Hypo- creales subtilisin-like proteases in known families and subfamilies. Click here for file \\ [http://www.biomedcentral.com/content/supplementary/1471- 2148-9-168-S9.doc]}

\section{Acknowledgements}

This research was supported by grants MAUX0127, CIOX0203 and MAUI03 to from the New Zealand Foundation for Research, Science and Technology (FRST) and the Royal Society of New Zealand Marsden fund (to Barry Scott), and grants EF-052366 I from the US National Science Foundation (to Christopher Schardl, Mark Farman and Bruce Roe) and 200535319-16141 from US Department of Agriculture National Research (to Christopher Schardl), for sequencing of the $E$. festucae genome. The authors thank Grant Hotter for his initial work on this project, Richard Johnson (AgResearch) for providing the N. Iolii kexB nucleotide sequence, and the $E$. festucae genome sequence consortium (University of Kentucky) for E. festucae E2368 genomic sequence. Special thanks to Bruce Roe (University of Oklahoma) for his assistance with sequencing the $E$. festucae genome. Michelle Bryant was the recipient of an AGMARDT Doctoral scholarship and supported by contract MAUI03.

\section{References}

I. Rawlings ND, Tolle DP, Barrett AJ: MEROPS: the peptidase database. Nucleic Acids Res 2004, 32:D I60-164.

2. Kraut J: Serine proteases: structure and mechanism of catalysis. Ann Rev Biochem 1977, 46:331-358.

3. Siezen RJ, Leunissen JA: Subtilases: the superfamily of subtilisinlike serine proteases. Protein Sci 1997, 6:50I-523.

4. $\mathrm{Hu} G$, St Leger RJ: A phylogenomic approach to reconstructing the diversification of serine proteases in fungi. J Evol Biol 2004, I7:|204-12|4.
5. Gunkel FA, Gassen HG: Proteinase K from Tritirachium album Limber. Characterization of the chromosomal gene and expression of the cDNA in Escherichia coli. Eur J Biochem 1989, I79:185-194.

6. Fabre E, Nicaud JM, Lopez MC, Gaillardin C: Role of the proregion in the production and secretion of the Yarrowia lipolytica alkaline extracellular protease. I Biol Chem |991, 266:3782-3790.

7. Takagi H, Koga M, Katsurada S, Yabuta $Y$, Shinde U, Inouye $M$, Nakamori S: Functional analysis of the propeptides of subtilisin $E$ and aqualysin $I$ as intramolecular chaperones. FEBS Lett 200I, 508:210-2I4.

8. St Leger RJ, Joshi L, Roberts DW: Adaptation of proteases and carbohydrates of saprophytic, phytopathogenic and entomopathogenic fungi to the requirements of their ecological niches. Microbiology 1997, 143:1983-1992.

9. Pinan-Lucarre B, Paoletti M, Dementhon K, Coulary-Salin B, Clave C: Autophagy is induced during cell death by incompatibility and is essential for differentiation in the filamentous fungus Podospora anserina. Mol Microbiol 2003, 47:321-333.

10. Takeshige K, Baba M, Tsuboi S, Noda T, Ohsumi Y: Autophagy in yeast demonstrated with proteinase-deficient mutants and conditions for its induction. I Cell Biol I992, I I 9:30 I-3 II.

II. Rogers DT, Saville D, Bussey H: Saccharomyces cerevisiae killer expression mutant kex 2 has altered secretory proteins and glycoproteins. Biochem Biophys Res Commun 1979, 90:187-193.

12. Conesa A, Punt PJ, van Luijk N, Hondel CA van den: The secretion pathway in filamentous fungi: a biotechnological view. Fungal Genet Biol 200I, 33:155-17I.

13. Faraco V, Palmieri G, Festa G, Monti M, Sannia G, Giardina P: A new subfamily of fungal subtilases: structural and functional analysis of a Pleurotus ostreatus member. Microbiology 2005, I 5 I:457-466.

14. Zhang J: Evolution by gene duplication: an update. Trends Ecol Evol 2003, 18:292-298.

15. Nei M, Rooney AP: Concerted and birth-and-death evolution of multigene families. Ann Rev Genet 2005, 39: I2I-I52.

16. Christensen MJ, Leuchtmann A, Rowan DD, Tapper BA: Taxonomy of Acremonium endophytes of tall fescue (Festuca arundinacea), meadow fescue ( $F$. pratensis) and perennial ryegrass (Lolium perenne). Mycol Res 1993, 97:1083-1092.

17. Moon CD, Tapper BA, Scott B: Identification of Epichloë endophytes in planta by a microsatellite-based PCR fingerprinting assay with automated analysis. Appl Environ Microbiol 1999, 65:1268-1279.

18. Moon CD, Scott B, Schard CL, Christensen MJ: The evolutionary origins of Epichloë endophytes from annual ryegrasses. Mycologia 2000, 92: I 103-III8.

19. Byrd AD, Schardl CL, Songlin PJ, Mogen KL, Siegel MR: The betatubulin gene of Epichloë typhina from perennial ryegrass (Lolium perenne). Curr Genet 1990, 18:347-354.

20. Möller EM, Bahnweg G, Sandermann H, Geiger HH: A simple and efficient protocol for isolation of high molecular weight DNA from filamentous fungi, fruit bodies, and infected plant tissues. Nucleic Acids Res 1992, 20:61 I5-6116.

21. Southern EM: Detection of specific sequences among DNA fragments separated by gel electrophoresis. J Mol Biol 1975, 98:503-517.

22. Young C, Itoh Y, Johnson R, Garthwaite I, Miles CO, Munday-Finch SC, Scott B: Paxilline-negative mutants of Penicillium paxilli generated by heterologous and homologous plasmid integration. Curr Genet 1998, 33:368-377.

23. Young CA, Bryant MK, Christensen MJ, Tapper BA, Bryan GT, Scott $B$ : Molecular cloning and genetic analysis of a symbiosisexpressed gene cluster for lolitrem biosynthesis from a mutualistic endophyte of perennial ryegrass. Mol Genet Genomics 2005, 274: 13-29.

24. Tanaka A, Tapper BA, Popay A, Parker EJ, Scott B: A symbiosis expressed non-ribosomal peptide synthetase from a mutualistic fungal endophyte of perennial ryegrass confers protection to the symbiotum from insect herbivory. Mol Microbiol 2005, 57:1036-1050.

25. Sambrook J, Russell DW: Molecular Cloning: a laboratory manual. New York: Cold Spring Harbor Laboratory Press; 2001. 
26. Sanger $F$, Nicklen $S$, Coulson AR: DNA sequencing with chainterminating inhibitors. Proc Natl Acad Sci (USA) 1977, 74:5463-5467.

27. Pearson WR, Lipman DJ: Improved tools for biological sequence comparison. Proc Natl Acad Sci U S A. 1988, 85(8):2444-2448.

28. Altschul SF, Gish W, Miller W, Myers EW, Lipman DJ: Basic local alignment search tool. J Mol Biol 1990, 21 5:403-4I0.

29. Altschul SF, Madden TL, Schaffer AA, Zhang J, Zhang Z, Miller W, Lipman D]: Gapped BLAST and PSI-BLAST: a new generation of protein database search programs. Nucleic Acids Res 1997, 25:3389-3402.

30. Bendtsen JD, Nielsen H, von Heijne G, Brunak S: Improved prediction of signal peptides: SignalP 3.0. J Mol Biol 2004, 340:783-795.

31. Thompson JD, Higgins DG, Gibson TJ: CLUSTAL W: improving the sensitivity of progressive multiple sequence alignment through sequence weighting, position-specific gap penalties and weight matrix choice. Nucleic Acids Res 1994, 22:4673-4680.

32. Tamura K, Dudley J, Nei M, Kumar S: MEGA4: Molecular Evolutionary Genetics Analysis (MEGA) software version 4.0. Mol Biol Evol 2007, 24:1596-1599.

33. Guindon S, Gascuel O: A simple, fast, and accurate algorithm to estimate large phylogenies by maximum likelihood. Systematic Biology 2003, 52:696-704.

34. Saitou N, Nei M: The neighbor-joining method: a new method for reconstructing phylogenetic trees. Mol Biol Evol 1987, 4:406-425.

35. Felsenstein J: Confidence limits on phylogenies: an approach using the bootstrap. Evolution 1985, 39:783-79I.

36. Zuckerkandl E, Pauling L: Evolutionary divergence and convergence in protease. In Evolving Genes and Proteins Edited by: Bryson V, Vogel HJ. New York: Academic Press; 1965:97-166.

37. Saeki K, Okuda M, Hatada Y, Kobayashi T, Ito S, Takami H, Horikoshi $\mathrm{K}$ : Novel oxidatively stable subtilisin-like serine proteases from alkaliphilic Bacillus spp.: enzymatic properties, sequences, and evolutionary relationships. Biochem Biophys Res Commun 2000, 279:313-319.

38. McGill MK: Cloning and characterisation of two subtilisin-like protease genes from Neotyphodium lolii. In M. Sc Palmerston North, New Zealand: Massey University; 2000.

39. Bryant MK: Functional analysis of genes encoding hydrolytic enzymes in the interaction of Epichloë festucae with perennial ryegrass. In PhD Palmerston North, New Zealand: Massey University; 2005.

40. Reddy PV, Lam CK, Belanger FC: Mutualistic fungal endophytes express a proteinase that is homologous to proteases suspected to be important in fungal pathogenicity. Plant Physiol 1996, III:1209-1218.

41. Bryant MK, May KJ, Bryan GT, Scott B: Functional analysis of a $\beta$ I,6-glucanase gene from the grass endophytic fungus Epichloë festucae. Fungal Genet Biol 2007, 44:808-8I7.

42. Johnson L], Johnson RD, Schardl CL, Panaccione DG: Identification of differentially expressed genes in the mutualistic association of tall fescue with Neotyphodium coenophialum. Physiol Mol Plant Pathol 2003, 63:305-317.

43. Luo X, Hofmann K: The protease-associated domain: a homology domain associated with multiple classes of proteases. Trends Biochem Sci 200I, 26:147-I48.

44. Brown CK, Gu ZY, Matsuka YV, Purushothaman SS, Winter LA, Cleary PP, Olmsted SB, Ohlendorf DH, Earhart CA: Structure of the streptococcal cell wall C5a peptidase. Proc Natl Acad Sci (USA) 2005, 102:18391-18396.

45. Venancio EJ, Daher BS, Andrade RV, Soares CM, Pereira IS, Felipe MS: The kex2 gene from the dimorphic and human pathogenic fungus Paracoccidioides brasiliensis. Yeast 2002, 19:|22|-|23|.

46. Wilcox CA, Fuller RS: Posttranslational processing of the prohormone-cleaving Kex2 protease in the Saccharomyces cerevisiae secretory pathway. I Cell Biol I99I, I I 5:297-307.

47. Saeki K, Ozaki K, Kobayashi T, Ito S: Detergent alkaline proteases: enzymatic properties, genes, and crystal structures. J Biosci Bioeng 2007, I 03:50I-508.

48. Nielsen CB, Friedman B, Birren B, Burge CB, Galagan JE: Patterns of intron gain and loss in fungi. PLoS Biol 2004, 2:e422.

49. Babenko VN, Rogozin IB, Mekhedov SL, Koonin EV: Prevalence of intron gain over intron loss in the evolution of paralogous gene families. Nucleic Acids Res 2004, 32:3724-3733.
50. Wang C, Typas MA, Butt TM: Phylogenetic and exon-intron structure analysis of fungal subtilisins: support for a mixed model of intron evolution. J Mol Evol 2005, 60:238-246.

5I. Hamer L, Pan H, Adachi K, Orbach MJ, Page A, Ramamurthy L, Woessner JP: Regions of microsynteny in Magnaporthe grisea and Neurospora crassa. Fungal Genet Biol 200I, 33:137-I43.

52. Eaton C], Jourdain I, Foster SJ, Hyams JS, Scott B: Functional analysis of a fungal stress-activated MAP kinase. Curr Genet 2008, 53:163-174.

53. St Leger R, Joshi L, Bidochka MJ, Roberts DW: Construction of an improved mycoinsecticide overexpressing a toxic protease. Proc Natl Acad Sci U S A. 1996, 93( ( 3):6349-6354.

54. Bagga S, Hu G, Screen SE, St Leger RJ: Reconstructing the diversification of subtilisins in the pathogenic fungus Metarhizium anisopliae. Gene 2004, 324:159-169.

55. Li J, Mahajan A, Tsai MD: Ankyrin repeat: a unique motif mediating protein-protein interactions. Biochemistry 2006 , 45: $15168-15178$
Publish with Bio Med Central and every scientist can read your work free of charge

"BioMed Central will be the most significant development for disseminating the results of biomedical research in our lifetime. "

Sir Paul Nurse, Cancer Research UK

Your research papers will be:

- available free of charge to the entire biomedical community

- peer reviewed and published immediately upon acceptance

- cited in PubMed and archived on PubMed Central

- yours - you keep the copyright
BioMedcentral 